\title{
Stratégies De Gestion Des Conflits Fonciers Intra Familiauxà Douafla Dans La Sous-Préfecture De Sinfra (Centre-Ouest Ivoirien)
}

\author{
Fallé Landry Yves \\ Socio-anthropologue (Enseignant-Chercheur) \\ Université Alassane Ouattara de Bouaké, Côte d'Ivoire
}

Doi: 10.19044/esj.2018.v14n2p23 URL:http://dx.doi.org/10.19044/esj.2018.v14n2p23

\begin{abstract}
Our study is focused on intra-family conflicts in the locality of Douafala at the sub-prefecture of Sinfra in the west center of Cote d'Ivoire. It has helped us to understand and analyze the causes of intra-family conflicts within the families of Douafla and the processes used in solving them. The argument is that intra-family land conflicts take many forms in the locality of Douafala and the actors use specific techniques to resolve these conflicts. The study was guided by the main question: What are the intra-family land conflicts existing in Douafla which justifies the land conflicts existing within the family unit? To answer this question, we have developed three hypotheses from the three specific objectives. Indeed, the first objective was to identify intra-family conflicts in Douafla. The second objective was to analyze the intra-family conflicts and the adopted (customary and administrative) strategies of this conflict. Finally, the last objective was to analyze the procedures adopted by the authorities in the resolution of this conflict. At the end of our analysis, we stated that land in all its forms generates conflicts that are sometimes adjustable. Land conflicts, therefore, arise due to generosity in the past and the lack of paper document of the land.
\end{abstract}

Keywords: Conflicts, land, intra-family, strategies, management, Douafla, Sinfra

\section{Résumé}

Notre étude a porté sur les conflits intra familiaux dans la localité de Douafala dans la sous-préfecture de Sinfra au centre ouest de la Côte d'ivoire. Il s'agissait pour nous d'analyser les causes des conflits intrafamiliaux et les procédés utilisés pour résoudre ces conflits au sein des familles de Douafla. L'argument est que les conflits fonciers intrafamiliaux prennent plusieurs formes dans la localité de Douafala et que les acteurs utilisent des techniques 
spécifiques pour résoudre ces conflits. L'étude était guidée par la question principale: Quels sont les conflits fonciers intrafamiliaux existant à Douafla qui justifient les conflits fonciers existant au sein de la cellule familiale? Pour répondre à cette question, nous avons développé trois hypothèses à partir des trois objectifs spécifiques. En effet, le premier objectif était d'identifier les conflits intra-familiaux à Douafla. Le second objectif était d'analyser les conflits intra-familiaux et les stratégies adoptées (coutumières et administratives) de ce conflit. Enfin, le dernier objectif était d'analyser les procédures adoptées par les autorités dans la résolution de ce conflit. À la fin de notre analyse, nous avons déclaré que le foncier sous toutes ses formes génère des conflits parfois ajustables. Les conflits fonciers sont donc dus à la générosité du passé et à l'absence de document papier sur la terre.

Mots-clés: Conflits, fonciers, intra familiaux, stratégies, gestion, Douafla, Sinfra

\section{Introduction}

La Côte d'Ivoire depuis son indépendance abasé son économie sur l'agriculture d'exportation (café, cacao, palmier à huile etc.). Cette situation a créé la ruée vers les terres fertiles du pays (sud, sud-ouest, sud est ouest.) pour la création des plantationsentrainant ainsi une anarchie dans l'occupation des parcelles de terreet par ricochetdes conflits fonciers. Ces conflits qui se présentent sous plusieurs formes opposent plusieurs acteurs (éleveurs/agriculteurs, les allochtones/autochtones, autochtones et allogènes (Fallé, 2014). A côté de ceux-ci, ils existent des conflits peu médiatiser au sein de la cellule familiale (les conflits intra et inter familiaux, intergénérationnelles etc.).

La localité de Douafla n'est pas en marge de cette situation conflictuelle intra familiale sur plusieurs parcelles de terres de la localité.C'est donc dans ce contexte précis que va s'effectuer notre étude.Alors quels sont les formes des conflits fonciers existants à Douafla ? Comment ces conflits fonciers se manifestent ils ? Quelles stratégies les familles adoptent-elles pour gérer ces conflits fonciers ?L'objectif général de cette étude est de comprendre les conflits fonciers intrafamiliaux et les stratégies de gestion. Cet objectif général va être soutenu par deux objectifs spécifiques qui vont de l'identification des conflits intrafamiliaux, aux stratégies adoptées des conflits, à l'analyse des procédures adoptées par les autorités (coutumières, administratives) dans la résolution des conflits.

La thèse soutenue est la suivante : les conflits fonciers intrafamiliaux se présentent sous plusieurs formes dans la localité de Douafala et les acteurs utilisent des techniques particulières pour résoudre ces conflits. 
Les constats qui sous-tendent cette étude sont de deux ordres: (I) l'absence de documents administratifs lors des transactions foncières; (II) la marginalisation de la femme dans le système d'héritage foncier. Ces éléments contribuent à l'exacerbation des conflits intra familiaux à Douafla.

En effet, les conflits intrafamiliaux naissent du manque des papiers administratifs dans le processus d'acquisition de terres. Plusieurs de propriétaires terriens n'ont pas de certificats fonciers ou titres fonciers. Cette absence de documents est dû au fait que les transactions foncières se faisaent par échange ou «troc ». Les parents vendaient les champs par échange avec des éléments à valeurs symboliques (boisson, cola) ou cette vente se faisait sous contrat appelé "ZEPA». En effet, lesristournes de ce contrat (zépa) pouvaitse chiffrer en nature, c'est-à-dire les parties prenantes se partagent équitablement la récolte ou quelques rare fois en numéraire.Egalement la vente de terre par le père garant de la propriété foncière familiale dans le passé sans documents précis (certificat de vente, titre fonciers, etc.) est souvent remise en cause par les nouvelles générations qui entraine des conflits. C'est aussi l'absence généralisée des documents au niveau des propriétés foncières familiales «nous n'avons aucun papier, c'est les terres de nos parents, c'est pour nous mais souvent nos cousins et oncles disent que c'est pour eux, or c'est notre père qui nous a donné. »M. TK (chef de famille). Le système d'héritage au niveau du foncier rural exclu toute transmission des terres à unefemme. A l'intérieur même du groupe détenteur de droits coutumiers « conformes aux traditions » à Douafla, les femmes sont mises à l'écart en ce qui concerne le contrôle, la gestion et l'appropriation des espaces fonciers. En ce sens lorsqu' un propriétaire terrien dont la cellule famille est composéeuniquement de filles comme descendantes directes, décède, son héritage foncier est légué à son frère direct. De ce fait, les enfants de sexe féminin sont automatiquement exclues de l'héritage foncier familial. La possibilité offerte aux femmes par la législation (code civil 20 et code foncier rural) d'avoir droit à la propriété foncière n'est que théorique (Koné, 2003).

Le refus du droit coutumier de reconnaitre à la femme héritière de son père défunt d'accéder à la terre est souvent source de conflits au sein de la famille, "La gestion des terres familiales apparait comme un "lieu» de tensions, de conflits entre parents et enfants, entre aînés et cadets » (Bologo, 2004). On assiste à une remise en cause ou à un rejet des lois coutumières pour poursuivre «l'affaire en justice » qui débouche sur un conflit intra familial.

\section{Matériel et Méthodes}

Cette étude s'inscrit dans le cadre d'une enquête qualitative principalement dans une étude de de cas. Elle nous permet de développer une analyse approfondie sur un groupe sociale donné pris dans son contexte (Alvoro, 1997). Plus singulièrement, elle renvoie dans le cadre de cette étude 
à une analyse compréhensive dans les conflits intrafamiliaux observés et des stratégies de gestion des conflits dans le contexte familial. Au cour de cette analyse se trouve la volonté manifeste d'identifier les conflits et les stratégies de gestion dans la cellule intra familiale dans la localité de Douafla.L'observation directe qui s'est faite à partir d'une grille d'observation à travers les réalités observées sur le terrain, elle a permis de savoir qu'il y a des conflits intrafamiliaux existants dans la localité de Douafla . L'enquête par entretien «individuel» constitue dans la recherche en sciences sociales un processus de construction de sens, une interrelation de production de discours qui engage le chercheur et l'enquêté (Quivy \& Campenhoudt, 1995). Dans le cadre de cette étude, nous avons opté pour l'entretien semi directif. Cette technique a donné une liberté d'expression dans les échanges tout en orientant leurs discours afin d'obtenir de nombreuses informations sur les conflits. Les guides d'entretien nous ont servi de fil conducteur dans le processus de collecte des données de terrain. Nous avons dressé les questions sur les thèmes en rapport avec notre sujet, à partir desquels se sont déroulés les entretiens avec les enquêtées.

Pour cette étude nous avons choisi comme population cible: les familles, les chefs et les autorités locales de la Marahoué résidant au quartier Douafla. L'échantillon principal retenu pour cette étude comprend au total 10 personnes répondant aux critères de sélection préétablis. A cet effet, nous avons interrogé 3 responsables administratifs 5 familles, 2 chefs coutumiers.

Tableau 1. Nombre des personnes interrogées

\begin{tabular}{|c|c|}
\hline Personnes interrogées & Nombres \\
\hline Personnels administratifs & 03 \\
\hline Chefs coutumiers & 02 \\
\hline Familles & 05 \\
\hline Total & 10 \\
\hline
\end{tabular}

Source: Enquête personnelle

Le champ sociologique consiste à préciser les caractéristiques des populations à étudier en donnant toutefois la raison de leur implication dans l'étude. Les populations étudiées sont des acteurs sociaux. S'agissant de notre thème d'étude nous avons choisi les familles du quartier Douafla ainsi que les autorités locales (le préfet, le maire, le sous-préfet, chef du service foncier etc.) et les chefs coutumiers (chef du village, chef de tribu) pour mener nos enquêtes afin de mieux cerner notre thème dans le milieu d'étude.

L'analyse stratégiquedéveloppée par Crozier et Friedbergqui a été mobilisé dans le cadre de cette étude.Les stratégies se rapportant aux acteurs sont adoptées en vue de gérer les conflits fonciers dont ils sont confrontés au quotidien à Douafla. Ainsi, elles s'appréhendent de façon concrète à travers les convocations et rencontres incessantes chez les différents chefs coutumiers, chef de terre et les plaintes au niveau du Ministère de l'agriculture 
de Sinfra. Par ailleurs, les stratégies mises en exergue par les acteurs dans la gestion des problèmes fonciers sont diverses. Ainsi, quel que soit les stratégies adoptées par les acteurs, elles visent à trouver une solution au profit de l'un des acteurs ou une solution conciliatrice. Egalement, elle donne une construction des règles à partir des marges et manœuvres des acteurs concernés et d'analyser le comportement des acteurs à travers les enjeux du foncier. Et l'individualisme méthodologique, qui est une théorie wébérienne ramenant l'explication de la réalité sociale aux actions individuelles en vue de la comprendre, en se mettant à la place des acteurs; de leurs actions individuelles. Raymond Boudon sensible à cette réalité affirmait que : "pour comprendre une réalité sociale, il convient de reconstituer les motivations des individus ; d'appréhender la réalité sociale comme l'agrégation c'est-à-dire la somme des comportements individuels des acteurs ». En ce qui concerne notre réalité sociale qu'est les conflits fonciers, le comportement des individus, leurs sources de motivation à s'impliquer dans des conflits est notre principal point d'appui à introduire dans notre travail cette théorie d'acteur individuel. Ainsi, il s'agira pour nous de récolter le point de vu des acteurs dans l'implication de ce conflit intrafamiliaux et les stratégies adoptées dans sa gestion.

\section{Résultats}

Douafla est un quartier de la ville de Sinfra qui est située dans le centre ouest de la Côte d'ivoire. Le département de Sinfra est situé dans la zone forestière et fait partie des trois départements de la région de la Marahoué (Bouaflé, Sinfra, Zénoula). 


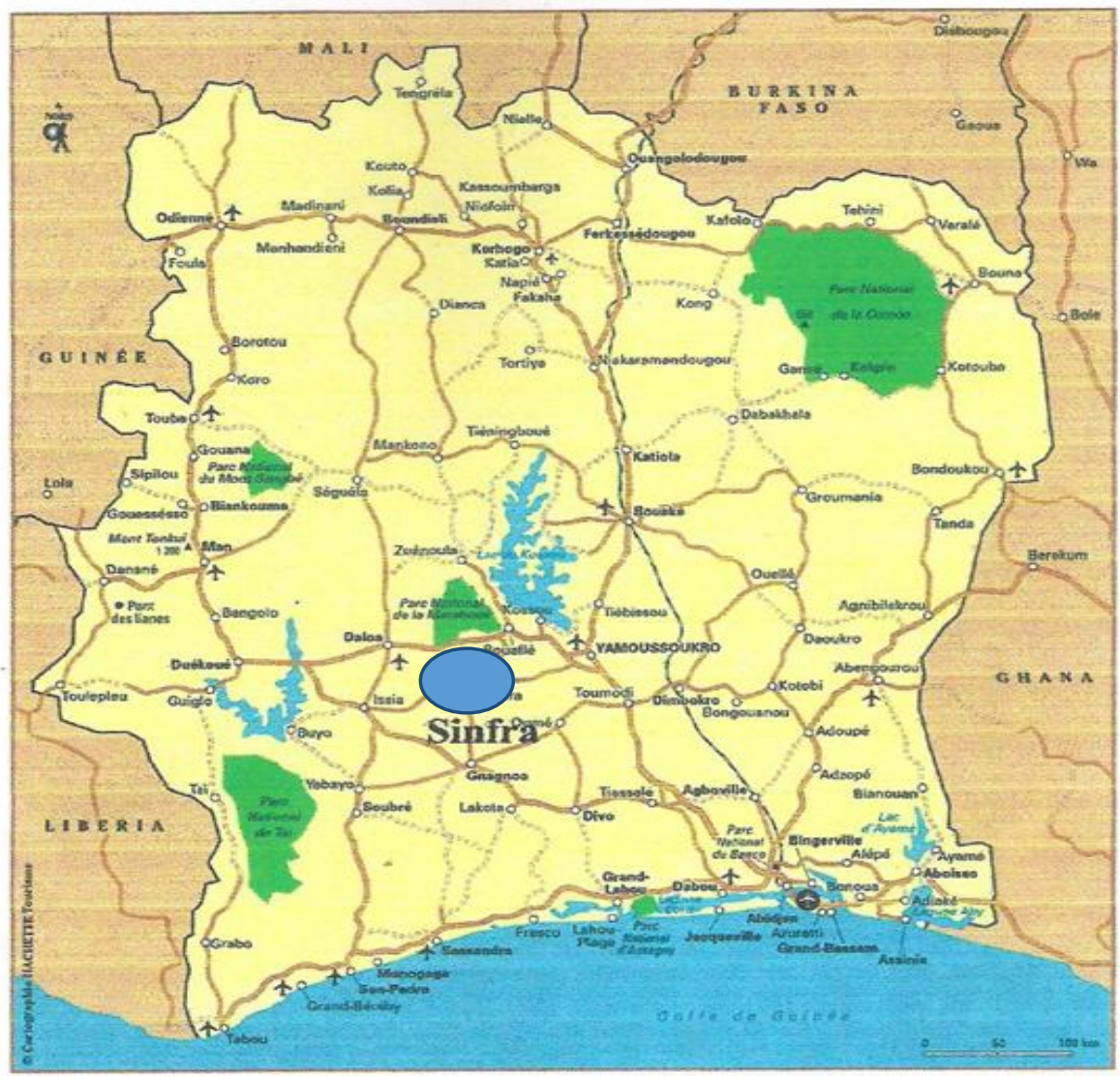

Légende: $\longrightarrow$ la ville de Sinfra

Source: Mairie de Sinfra

Douafla est un quartier de la localité de Sinfra et en même temps un village Gouro. Situé à l'ouest de la ville, il est limité au sud par le quartier Lycée, au nord par les villages de Blontifla, et Djamadji, et à l'Est par le quartier Résidentiel. C'est un quartier essentiellement composé d'autochtones. La subdivision du territoire du département de Sinfra est faite sur la base de tribus et sont composées comme suit: les Sian, Nanan, Bindin, Vinan, N'goye et Progouri. Cette subdivision tribale à une importance véritable en telle enseigne qu'elle dépeint dans la dénomination du nom de la ville "SINFRA". Cette appellation viendrait du nom proverbe de la tribu originelle "SIAN". Sinfra est donc en réalité "Sinfla", terroir des Sian, village des « Sians » qui a subi une déformation du fait des colons. "SIAN" ou "Kinnin" (en Gouro) est une nasse; c'est à dire un instrument traditionnel de pêche. Regroupés en général dans les villages, les Gouro de Sinfra pratiquent l'agriculture. Ils produisent le cacao, le café et dans une certaine mesure le coton d'où l'importance e la terre dans leur quotidien. Dans notre étude nous nous 
intéressons aux autochtones Gouro particulièrement à ceux du quartier de Douafla de Sinfra.

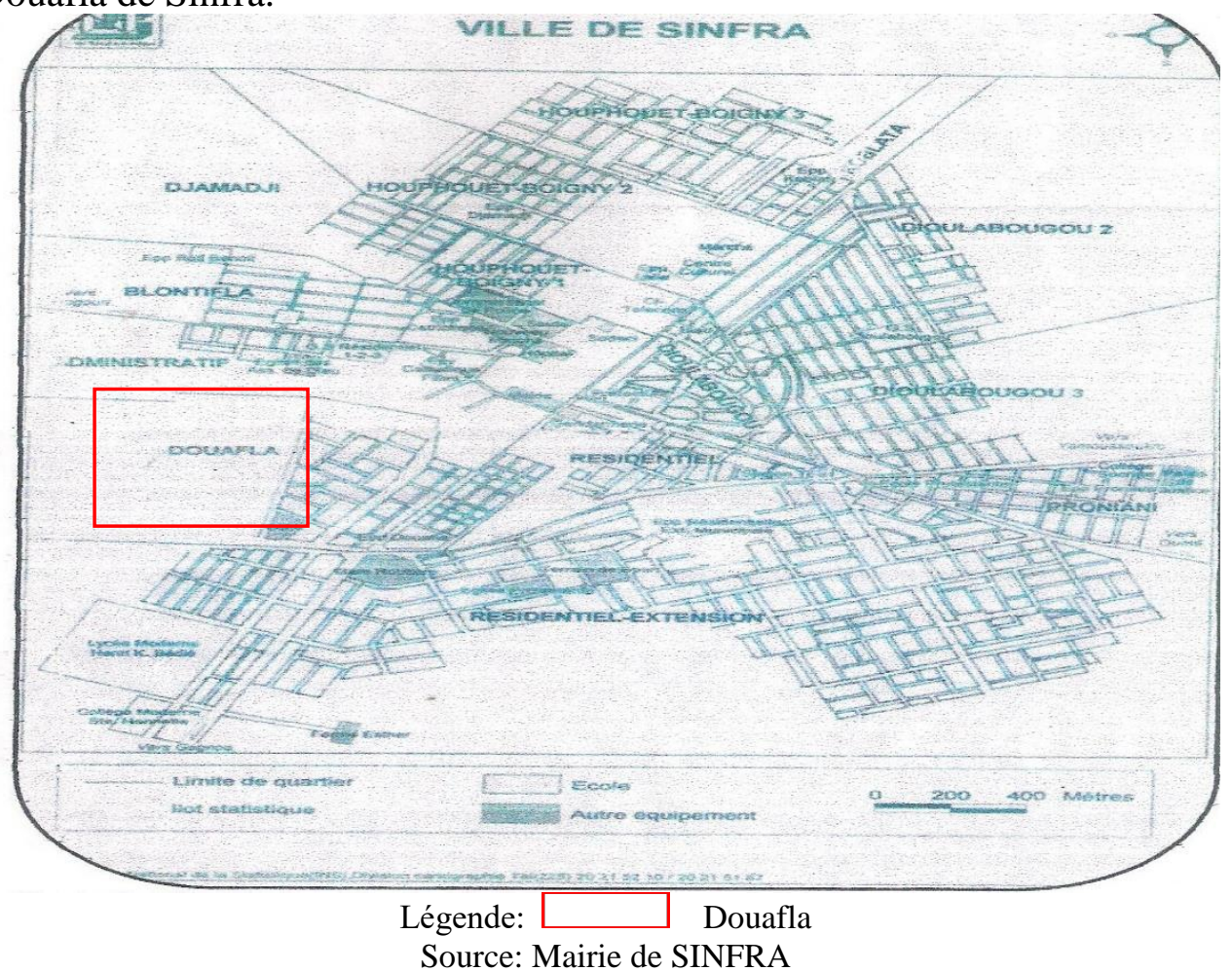

\section{Présentation des Conflits Intrafamiliaux et Leurs Manifestations à Douafla}

A Douafla, village caractérisé par une très forte pression foncière, on observe la présence de nombreux conflits intra familiaux liés à la terre. Ils se présentent sous plusieurs formes. Les conflits fonciers liés à l'héritage, ceux liés à l'insuffisance des terres familiales et enfin les conflits liés à la vente illégale de terre.

Tableau 2. Récapitulatif des conflits intra familiaux enregistrés

\begin{tabular}{|c|c|}
\hline Origine & Nombre \\
\hline Les conflits fonciers liés à l'héritage & 1 \\
\hline Les conflits fonciers liés à la raréfaction des terres familiales & 1 \\
\hline les conflits liés à la vente illégale de terre & 1 \\
\hline
\end{tabular}

Source : Enquête personnelle

\section{Les Manifestations des Conflits Fonciersliés à L'héritage}

Les conflitsfonciers à Douafla montrent que les conflits les plus violents sont ceux liés à l'héritage entre frères, sœurs ou membre de la même famille au sens élargi. Ils sont si violents qu'ils occasionnent dans certains cas des fragmentations de la cellule familiale. 


\section{Conflit Entre Frères}

$1^{\mathrm{er}}$ Cas: « un jeune frère qui allait à l'école était en classe de première mais à cause de l'héritage il a quitté les bancs pour venir rien que pour lutter le bien de la famille, et dans cette lutte il a tout fait pour éliminer sa sœur croyant que la part de sa grande sœur c'est-à-dire l'héritage de sa grande sour allait lui revenir » (propos de la famille T.V.L.O)

2eme Cas (Propos de Madame D): «c'est mon mari qui devait être chef de terre, mais son frère est venu et à récupérer tout ce qui était terrain, plantation. Mon mari aussi ne dit rien. "»

\section{Conflit Entre Oncle et Nièce}

3eme Cas: « mon oncle ayant été chassé de sa maison à Abidjan, je lui ai demandé de rester dans la cour que j'avais construit pour mon père. Et puis, et puis lorsque mon oncle décéda sa femme refusa que je m'installe dans la maison, disant que la maison appartient à son mari » (propos d'anonymat).

Ces différents faits montrent l'origine de certains conflits fonciers intrafamiliaux, l'attitude de certains acteurs pour accéder ou récupérer des biens fonciers.

\section{Les Manifestations des Conflits Liés à la Raréfaction de la Terre à Douafla}

Ils sont dus à la générosité et aux contrats passés entre les propriétaires terriens et des particuliers ou des groupements des personnes dans des années antérieures. A l'époque, Il y avait abondances de terres, et l'on donnait au premier arrivant des lopins de terres pour pratiquer l'agriculture. Cette charité a poussé les parents d'alors a octroyé des terres par pure générosité, "A cause de notre générosité, actuellement là pour que nos enfants et nos petits-enfants ait la terre pour cultiver c'est difficile et sa sera difficile, tout est occupé et aujourd'hui on a ce problème là et c'est ce qui engendre ce genre de conflit ». (Propos du Chef du village de Douafla). "Ici à Douafla mon grand frère qui est là,il est le petit frère à mon papa et lui aussi a des enfants, comme mon papa lui-même, comme moi si d'aventure il a fait des enfants et il en a qui sont allés à l'école eux ils travaillent là-bas. Mais ceux qui sont au village quand ils viennent là, ils disent ah! Comme je n'ai pas eu de bureau la bas (en ville), je veux aller au champ, au champ là où il va avoir la terre pour pourvoir cultiver, et l'enfant va avoir quelque chose pour se mettre sous la dent.Quand il va la bas il trouve que le champ de son papa est insuffisant. " (Propos du Chef $d u$ Village). La rareté des terres est un phénomène réel à Douafla. Les jeunes autochtones déscolarisés, au chômage, voulant retourner à la terre dans le but d'avoir une activitérémunératrice se trouvent confronter à un manque de terre. La quasi-totalité des terres arables sont occupées, c'est-à-dire $98 \%$ des terres sont déjà mises en valeur. L'existence de parcelles cultivables est 
peu probable : "Il n'y a plus de parcelle, il n'y a plus de plantation,il n'y a plus de forêt ou nos petit enfants, nos arrières petits enfants vont quand même un jour venir s'asseoir, tout est déjà occupé. » (K.S)

L'absence de terre a donné une valeur marchande au foncier, ce qui se donnait facilement à travers des objets symboliques sous une simple parole, est monétarisée, "Une petite parcelle ou quelqu'un va venir, le monsieur comme on lui avait donné, bon par exemple s'il a payé,s 'il a donné au moins 20.000 mille francs,je dis avant gracieusement on donnait,voilà à cause de 20.000 mille, une bouteille de liqueur ou 5000 on peut lui donner (2ha ou 3ha),alors que maintenant là tout ceux qui avait faitça,ils sont tous décédées,ils sont parti ».(notable gouro). La remise en cause par les nouvelles générations des accords passés dans le but de réattribuer la paternité d'un territoire famille dans le but de faciliter son accès. "La nouvelle génération maintenant là chacun a envie d'avoir une petite parcelle pour faire son champ de cacao, son champ de café, y a n'en pas vous voyez donc il est obligé de se défendre, en disantque ah ici là, c'est pour mon papa mais pourquoi tu veux t'asseoir comme ça? Etpuis tu as (2 ha) or moi je n'ai même pas (1/2ha), c'est là que les conflits commencent» (Proposdu Chef du village de Douafla).

\section{Les Manifestations des Conflits Liés à la Vente Illégale de Terre}

Ces conflits sont beaucoup perceptibles entre cousins appartenant à une même famille. La vente de terre appartenant aux membres de la grande famille par un cousin ou frère directe «Les gens de la même famille, ils ont le même grand père, avant Douafla c'était des arbres mais au fur et à mesure que le temps à évoluer, on a fait des lotissements jusqu'à s'est arrivé au carrefour la bas tout ça c'est Douafla, même après le carrefour c'est Douafla. C'était la brousse, champ de café, de cacao ainsi de suite. Donc le champ de caféier qui était à côtéici, appartenait à un frère qui était là comme ils sont parentés;son papa et celui qui a vendu la parcelle était de même père, ils ont même grand père, ils n'ont pas la même grand-mère,parce que chez nous les Gouro on se marie a beaucoup de femmes, moi-même j'ai deux femmesvoilà !donc le vrai Gouro là s'il n'a rien c'est deux. Donc chacun à sa maman,donc c'est comme ça, chacun à son champ de caféier qui est rentré ici. Comme la ville a dépassé les champs, c'est devenu des lots. Mais il y avait des parcelles qui était là, il sait que c'est le champ de son papa. Donc les enfants sont venus, comme c'est devenu des lots il a vendu, il sait que c'est le champ de son papa, alors que ce n'était pas le champ à son papa, mais le champ du grand frère a son papa. On a un vieux âgé là-bas c'est lui qui a dit non sa vous appartient dans la famille ; le monsieur qui est là c'était son papa qui avait son champ de caféier ici. Ce que tu as vendu là !c'est familiale mais l'intéresse lui-même c'est x "(propos de Monsieur K.B.). 
Plusieurs structures et acteurs interviennent dans le cadre de la gestion des conflits fonciers intrafamiliaux. Il s'agit principalement des chefs coutumiers (traditionnels), des autorités administratives.

\section{La Procédure Par Conciliation dans la Gestion des Conflits}

La procédure de gestion par conciliation, est la procédure par laquelle les autorités coutumières parviennent à la résolution d'un conflit avec l'accord des parties, C'est-à-dire l'accusé et le plaignant. Par exemple: "Quand un problème naît entre deux personnes (frère et sœur, oncle et neveu,), on s'assoit, on essaie de se comprendre, on essaie de s'expliquer devant les personnes âgées eux ils apportent leur expertise, leur connaissance, leur témoignage, si ça ne va pas on essaie d'évoluer, si ça ne va pas on avise les chefs coutumiers qui essaie de régler cette histoire à l'amiable.» (Monsieur K.B.H)

Les chefs de village ou de tribu sont compétents pour concilier les ressortissants du village ou de la tribu, partie à un conflit. Ils sont le plus souvent assistés de l'autorité religieuse lors de la conciliation. En cas de réussite de la conciliation, le chef établi un procès-verbal de conciliation dans le cas contraire le chef de village ou de tribu établit un procès-verbal de nonconciliation et fais recours au chef de canton. Les chefs de canton avec l'aide des notables procèdent de la même manière que le chef de village et de tribu, en cas d'échec il est établi un procès-verbal de non-conciliation et les parties sont renvoyées devant le Tribunal d'Instance, juridiction siégeant au niveau départemental.

Figure 1. Schéma de règlement des conflits par conciliation

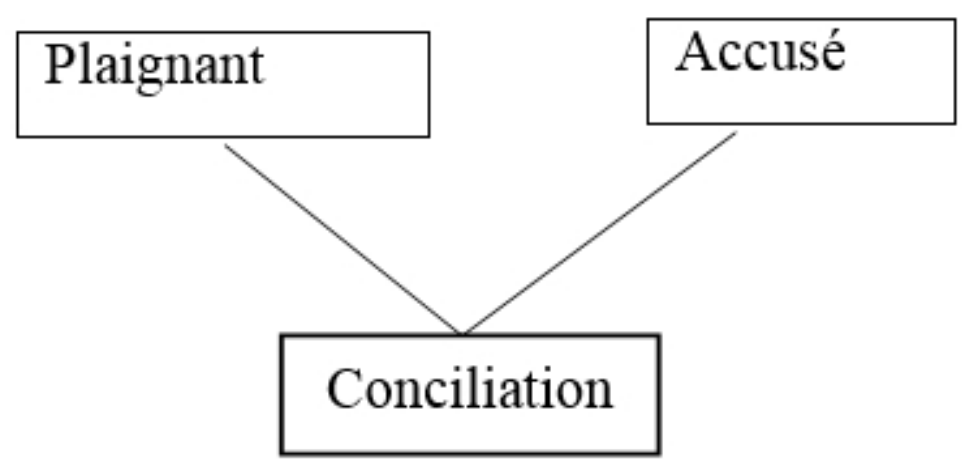

Source : Enquête personnelle

\section{La Procédure Administrative et Judiciaire}

La procédure de gestion administrative refuse souvent la gestion des conflits mais quand elle est prise en compte et ramène généralement les protagonistes au comité de gestion locales (chef de village, notables, chef de 
famille etc.). Elle se fait souvent pas conciliation au niveau du cadre familial ou finit quelquefois à la justice, si les parties prenantesn'arriventpas à trouver un terrain d'entente. En réalité, les autorités administratives de par la législation en vigueur ne disposent pas, toutefois de pouvoir de conciliation, c'est pourquoi les lois administratives en vigueur ne donnent quecette compétence qu'aux autorités coutumières. Tout ceci afin d'éviter la procédure judiciaire car à leur niveau la conciliation n'est pas prise en compte c'est ainsi qu'ils font régulièrement recours aux chefs coutumiers.

La procédure de gestion judiciaire quantà elle, en matière de conciliation et de jugement des conflits conformément aux dispositions applicables, est compétente. Elles interprètent les lois et les coutumes et sont chargés de leur application. En matière coutumière, le tribunal d'Instance est la juridiction de premier degré. La partie qui se sent lésée suite à une décision rendue par ce dernier peut faire appel devant le tribunal de grande instance, juridiction d'appel des décisions rendues en matière coutumière par les tribunaux d'instance.

En sommes, toutes ces procédures en passant par les chefs coutumiers, les autorités administratives et judiciaires concourent à gérer les conflits fonciers intrafamiliaux dans le but d'un règlement définitif des conflits.

Tableau 3. Représentation des étapes de gestion des conflits

\begin{tabular}{|c|c|c|}
\hline $\begin{array}{c}\text { Etape du } \\
\text { règlement de } \\
\text { conflit }\end{array}$ & Niveau & Acteurs \\
\hline $\begin{array}{c}\text { Règlement à } \\
\text { l'amiable }\end{array}$ & Les parties prenantes (accusé et plaignant) & $\begin{array}{c}\text { Les parties prenantes(accusé et } \\
\text { plaignant) }\end{array}$ \\
\hline Conciliation & Chef du village, de tribu ou de canton & $\begin{array}{c}\text { Chef du village, de tribu ou de } \\
\text { canton }\end{array}$ \\
\hline Administrative & $\begin{array}{c}\text { Préfet, sous-préfet, chef départemental de } \\
\text { l'agriculture (Directeur départemental de } \\
\text { l'agriculture), }\end{array}$ & $\begin{array}{c}\text { Préfet, sous-préfet, chef } \\
\text { départemental del'agriculture } \\
\text { (Directeur départemental de } \\
\text { l'agriculture), }\end{array}$ \\
\hline Judiciaire & Commissariat, police, gendarmerie & $\begin{array}{c}\text { Commissariat, police, } \\
\text { gendarmerie }\end{array}$ \\
\hline
\end{tabular}

Source : Enquête personnelle

\section{Discussion}

Dans cette partie, il s'agira, tout en rappelant les principaux résultats de l'étude, de les mettre en perspective avec les écrits antérieurs à la question de recherche à savoir les stratégies de gestion des conflits fonciers intrafamiliaux. Dans cette variabilité d'approches qui caractérise les écrits sur les stratégies de gestion de conflit intrafamiliaux certains auteurs se sont penchés sur la question d'héritage qui crée des divisions au sein de la famille. C'est dans cette optique que COLIN précise que, les tensions intrafamiliales autour de l'héritage peuvent se transformer en conflits intercommunautaires. Cet auteur fait une ouverture sur le sujet en affirmant que le problème 
d'héritage créant des conflits au sein de la famille peut s'étendre aussi en dehors de la famille. En occurrence, les transactions foncières peuvent, en effet, conduire à de fortes tensions intrafamiliales et intergénérationnelles, lorsque elles sont contestées la légitimité de la cession en tant que telle, ou la légitimité du «vendeur » (avec tout l'enjeu du contrôle ou du partage de la rente). Certains conflits post-héritage apparaissent ainsi, entre les jeunes arrivés à maturité et les «surveillants » de terre (oncle ou frère du père défunt) qui avaient la garde de l'héritage, lorsque l'héritier, devenu majeur ou rentré de migration, estime que le surveillant a vendu abusivement de la terre (Chauveau et al., 2006: 9). En effet, les ventes illicites liées à la terre créent des conflits intrafamiliaux. Aussi comme pouvait affirmer Jacob (2006), lorsque la communauté principale est la cellule familiale, il ne peut qu'y avoir des tensions au sein d'elle car les différentes générations au sein des familles ne se succèdent par à proprement parler, mais sont en perpétuelles position de chevauchement. Ce phénomène est source de tension qui s'exprime ou non sous forme de conflit ouvert à l'intérieur de la famille et qui créent parfois des divisions sans fin. Alors, Bologo Eric soutient qu'à Séguéré, la gestion intrafamiliale des droits fonciers porte sur un patrimoine foncier c'est-à-dire que le chef de famille est le seul détenteur des droits coutumiers d'appropriation et d'administration. Pour eux, l'attribution des droits d'usage au sein de la famille est fonction du statut, de la position sociale des individus dans le groupe, l'ordre de priorité des ayant droits est fait comme suit : les héritiers, les hommes mariés, les hommes célibataires en âge de se marier. Pour lui c'est par droit d'aînesse que se fait le partage d'héritage. Contrairement à des études menées dans le Centre Ouest du Burkina, (Jacob, 2001) et dans la zone de plantation ivoirienne (Colin, 2004) semblent montrer que la gestion intrafamiliale des droits ne fait pas apparaitre des conflits, sauf très rares exceptions. Ce qui sous-entend que la gestion des conflits ne sont toujours par source de division et que la plupart des disputes opposent des membres d'un même groupe familial, c'est-à-dire non pas pour obtenir une garantie, comme une sécurité foncière conçue comme bien privé mais au contraire, pour réaffirmer sa valeur en tant que bien commun (flexibilité maintenue de l'accès à la ressource pour l'ensemble des ayants droit). Des points de vue émergents entre celle de Colin et al. (2004) et la mienne. Hormis la réaffirmation de la valeur communautaire du bien foncier, nous nous inscrivons en faux contre le reste de son affirmation. De plus, ces études à l'instar de Colin et al. (2004), en ne relevant au niveau familial, que la dimension intrafamiliale au détriment de l'axe interfamilial, semblent minimiser leur ampleur. Elles semblent porter un regard réducteur sur les conflits fonciers. En définitive retenons que, les conflits dans la gestion des terres familiales entraînent assez souvent des conflits intercommunautaires. 
Mieux, nous estimons comme Fané (2009:68) que les questions foncières rurales constituent les vrais enjeux de l'évolution des sociétés africaines. Donc, l'explication des conflits qui y ont cours ne doit pas demeurer uniquement macrosociologique. Elle doit également s'inscrire dans un cadre microsociologique et spécifique, en l'occurrence celui de la famille.

\section{Conclusion}

Le travail de cette recherche que nous venons de conduire consiste à comprendre les stratégies de gestion foncière de conflits intrafamiliaux en Côte d'ivoire. Notre choix, pour la conduite de notre enquête de terrain s'est porté particulièrement sur les familles du village Douafla dans la souspréfecture de Sinfra, dans le centre ouest de la cote d'ivoire. Car l'ouest ivoirien, en général, connaît une forte récurrence de conflits fonciers. Ainsi cette étude a été guidée par la question principale : Quels sont les conflits fonciers intrafamiliaux existant à Douafla justifient-ils les conflits fonciers existant au sein de la cellule familiale?

Pour répondre à cette question nous avons élaboré trois hypothèses issues des trois objectifs spécifiques. En effet, le premier objectif a été d'identifier les conflits intrafamiliaux à Douafla. Le deuxième objectif était d'analyser les conflits intrafamiliaux et les stratégies adoptées (coutumière et administrative) de ceci. Enfin le dernier objectif était d'analyser les procédures adoptées par les autorités dans la résolution de ce conflit. Ces trois objectifs ont donné lieu aux hypothèses suivantes:

- Les conflits intrafamiliaux sont dus aux problèmes d'héritages.

- Les familles adoptent différentes résolutions des conflits intrafamiliaux à Douafla.

- Les autorités adoptent la procédure administrative pour résoudre ce type de conflit.

Au sortir de cette étude nous arrivons à de nombreuse conclusion. D'abord au niveau de notre principale hypothèse il ressort que les conflits sont dus aux problèmes d'héritage, nous avons vues qu'il y a des conflits entre oncle et neveu, entre frère et sœur, notre hypothèse a cependant montrer quelques insuffisances.

Ensuite, les deux autres hypothèses ont mis l'accent sur la gestion et les stratégies que les différents acteurs mettre en place pour gérer cette affaire foncière au sein de la famille.

Enfin, À ce niveau nos hypothèses ont confirmé nos différents constats généraux. Au terme de notre analyse, nous disons que le foncier sous toutes ses formes engendre des conflits qui sont parfois réglable. Les conflits fonciers sont donc pour eux, dues à leurs générosités dans le passé et à leur manque de papier. 


\section{References:}

\section{Ouvrages de méthodologie}

1. Alvaro, P. (1997). "Échantillonnage et recherche qualitative : essai théorique et méthodologique. Montréal, Gaëtan Morin, 405 pp.

2. Durkheim, E. (1983). Les règles de la méthode sociologique. Paris, PUF, $21^{\mathrm{e}}$ Edition, page 34.

3. Granai, G. (1967). « Techniques de l'enquête sociologique», in GURVITCH G. Traité desociologie. Tome premier, Paris, PUF.

4. Kouakou, B. J-P. (2011). Guide d'écriture bibliographique. Abidjan, Balafons.

5. N'DAP. (2015). Recherche et méthodologie en sciences sociales et humaines : réussir sa thèse, son mémoire de master ou professionnel, et son article, Paris, Le Harmattan.Paris, Dunod.

6. Quivy, R. \& Van Campenhoudt, L. (1995). Manuel de recherche en sciences sociale.

\section{OUVRAGES GENERAUX}

7. Babo, A., Droz, Y., Chauveau, J.-P., \& Mathieu, P. (1998).

8. Chauveau, J.-P. (2004). Conflit, crise de la ruralité et crise foncière en Côte d'Ivoire forestière: le " tutorat » entre autochtones et migrants au centre des tensions. Atelier CLAIMS, Mali.

9. Chauveau, J.-P. (2006). Les transferts coutumiers de droits entre autochtones et "étrangers ». Évolutions et enjeux actuels de la relation de " tutorat ». In CHAUVEAU, J.P., COLIN, J.Ph., LAVIGNE DELVILLE, Ph. et LE MEUR, P.-Y. Rapport final Programme de recherche européen CLAIMS. Londres : IIED.

10. Colin, J.-P. (2004). Droits fonciers et dimension intrafamiliale de la gestion foncière.

11. Coulibaly, A. (2006). Gestion des conflits fonciers dans le Nord ivoirien: Droits, autorités et procédures de règlement des conflits, Colloque internationale «Les frontières de la question foncière - At the frontier of land issues», Montpellier: IRD.Dynamiques et enjeux des conflits fonciers. in DELVILLE LAVIGNE, Ph. (Dir.).

12. Jacob, J.-P. (2001). Systèmes locaux de gestion des ressources naturelles et approches développementalistes : le cas du Gwendégué (centre ouest Burkina Faso).in Autrepart 19. Montpellier : IRD. pp. 133-153.

13. Koné, M. \& Kouamé, N. (2005a). Socio anthropologie de la famille en Afrique. Evolution des modèles en Côte d'Ivoire. Abidjan, Les Editions CERAP Note méthodologique pour une ethnographie économique de l'accès à la terre en Afrique. Montpellier: IRD.

\section{REVUES ET RAPPORTS}

14. Gillian Mathys \& Koen Vlassenroot (2016). «pas juste une question 
de terre » : litiges et conflits fonciers dans l'est du Congo.

15. Human Right Club (2013). "Cette terre est la richesse de ma famille ", Agir contre la dépossession de terre suite au conflit postélectoral en Côte d'Ivoire.

16. Rapport du Club UA-CI sur la situation du foncier rural en Côte d'Ivoire.

17. République du Niger Comité National du Code Rural Secrétariat Permanent du Code Rural

18. Revue Africaine de Sociologie, 7, (2), 2003, pp. 58-72.

19. Memoire et these

20. Kouakou Marius Roland (2014-2015). «Socio-histoire de la violence criminelle autour du foncier rural dans l'ouest ivoirien » (cas de Fengolo s/p de Duékoué).

21. Monsieur Zohoré Togba Gagui (2016). «Les Déterminants culturels et individuel des conflits fonciers interfamiliaux en milieu rural » : cas du département d'Issia. 